\title{
QUASILINEAR ELLIPTIC EQUATIONS WITH VMO COEFFICIENTS
}

\author{
DIAN K. PALAGACHEV
}

\begin{abstract}
Strong solvability and uniqueness in Sobolev space $W^{2, n}(\Omega)$ are proved for the Dirichlet problem

$$
\left\{\begin{array}{l}
a^{i j}(x, u) D_{i j} u+b(x, u, D u)=0 \text { a.e. } \Omega \\
u=\varphi \text { on } \partial \Omega .
\end{array}\right.
$$

It is assumed that the coefficients of the quasilinear elliptic operator satisfy Carathéodory's condition, the $a^{i j}$ 's are $V M O$ functions with respect to $x$, and structure conditions on $b$ are required. The main results are derived by means of the Aleksandrov-Pucci maximum principle and Leray-Schauder's fixed point theorem via a priori estimate for the $L^{2 n}$-norm of the gradient.
\end{abstract}

\section{INTRODUCTION}

The main purpose of the present article is to study strong solvability of the Dirichlet problem for quasilinear elliptic equations. More precisely, we deal with the equation

$$
\sum_{i, j=1}^{n} a^{i j}(x, u) D_{i j} u+b(x, u, D u)=0
$$

assuming $a^{i j}(x, z)$ and $b(x, z, p),(x, z, p) \in \Omega \times \mathbf{R} \times \mathbf{R}^{n}$, to be Carathéodory's functions. Our aim is to prove existence and uniqueness of a twice weakly differentiable function $u(x)$ satisfying $(0.1)$ almost everywhere in $\Omega \subset$ $\mathbf{R}^{n}$, with Dirichlet boundary data.

The classical boundary value problem for $(0.1)$ with sufficiently smooth coefficients and nonlinear term has been very well studied (cf. [GT]). Furthermore, assuming the principal coefficients $a^{i j}$ to be merely continuous functions and employing $L^{q}$-Schauder theory for linear elliptic operators, it is possible to derive suitable a priori bounds and therefore to prove various existence results. We refer to the papers by Tomi [To], Amann and Crandall [AC] (in the semilinear case $\left.a^{i j}=a^{i j}(x)\right)$ for the classical solvability, and to those by Troianiello [Tr] and Lieberman [L] in the case of weakly differentiable

Received by the editors July 7, 1994.

1991 Mathematics Subject Classification. Primary 35J65, 35B45.

Key words and phrases. Strong solution of elliptic PDE, VMO, a priori estimates, Aleksandrov-Pucci maximum principle, Leray-Schauder fixed point theorem.

The author was supported by the Italian National Research Council (C.N.R.).

(C) 1995 American Mathematical Society 
solutions. All these results assumed the nonlinear term $b(x, z, p)$ to grow at most quadratically in the gradient. On the other hand, there is a classical result due to Miranda [M] concerning $W^{2,2}$-solvability for semilinear operators with coefficients $a^{i j}(x) \in W^{1, n}$ and strictly sub-quadratic growth in $b(x, z, p)$ with respect to $p$.

Our goal here is to investigate Dirichlet's problem for $(0.1)$ weakening the assumptions on the behavior of the coefficients $a^{i j}(x, z)$ in the $x$ variables. Precisely we assume the coefficients to belong to the class of functions with vanishing mean oscillation ( $V M O$ ) locally uniformly in $z$. A natural background for these considerations is the recently developed $L^{q}$-Schauder theory of linear elliptic operators with $V M O \cap L^{\infty}$ coefficients (see [CFL1, CFL2]). It is easily seen that both cases $a^{i j} \in C^{0}$ or $a^{i j} \in W^{1, n}$ imply $a^{i j} \in V M O$ (cf. [CFL1, $\S 2])$.

The main tool in proving our existence result (Theorem 1.1) is the LeraySchauder fixed point theorem that reduces solvability of Dirichlet's problem to the establishment of a suitable a priori estimate for solutions of $(0.1)$ (in our case, the $W^{1,2 n}(\Omega)$-estimate). We start with $\|u\|_{L^{\infty}(\Omega)}$-bound that follows easily from the Aleksandrov-Pucci maximum principle. The second step in this procedure is to control modulus of continuity of $u(x)$. Indeed, Krylov-Safonov's Harnack inequality leads to an a priori bound for a suitable Hölder norm of $u$. In order to estimate the $L^{2 n}(\Omega)$ norm of the gradient $D u$, independently of $u$, we employ a method introduced by Tomi [To] and refined by Amann and Crandall [AC]. The idea is to imbed the problem into a family of Dirichlet problems depending on a parameter $\sigma$, with solutions $v_{\sigma}$ for $\sigma \in[0,1]$. If we are able to control the $W^{1,2 n}$-norm of the difference $v_{\sigma_{1}}-v_{\sigma_{2}}$ for sufficiently small $\left|\sigma_{1}-\sigma_{2}\right|$ then $\left\|D v_{\{\sigma=1\}}\right\|_{L^{2 n}(\Omega)}$ can be estimated in terms of $\left\|D v_{\{\sigma=0\}}\right\|_{L^{2 n}(\Omega)}$ after iteration. Thus the desired $L^{2 n}$-gradient bound for $u(x)=v_{\{\sigma=1\}}$ follows assuming in addition that we have unique solvability for the considered family. The crucial step in these investigations is ensured by the linear $L^{q}$-estimate for elliptic operators with $V M O$ coefficients due to Chiarenza, Frasca and Longo (see [CFL2]). Applying again that result, and bearing in mind the $W^{1,2 n}(\Omega)$ bound we had proved before, it is easy to estimate the $W^{2, n}$-norm of solution of $(0.1)$.

Let us point out that the approach used here implies strong solvability for (0.1) based merely on $L^{2 n}$-a priori (not $L^{\infty}$ !) estimate for the gradient. In other words, we are able to prove the existence of strong solution of $(0.1)$ without any requirements on $L^{q}(\Omega)$-integrability of $b(x, z, p)$ with respect to $x, q>$ $n$, as it was done in [Tr] and [L]. Indeed, if we assume more regularity on the data (cf. Corollaries 1.2 and 2.7) then the $L^{2 n}$-estimate for the gradient leads automatically to Hölder $C^{1,1-\frac{n}{q}}(\bar{\Omega})$-bound through $W^{2, q}$-estimate and Morrey's lemma.

Finally, uniqueness assertions (Theorem 1.4 and Corollary 1.5) are consequences of Aleksandrov-Pucci maximum principle assuming in addition $b(x, z, p)$ to be Lipschitz continuous with respect to $p$.

\section{BASIC ASSUMPTIONS AND MAIN RESULTS}

Let $\Omega$ be a bounded domain in $\mathbf{R}^{n}, n \geq 2$. The Sobolev space of $k$ times weakly differentiable functions whose derivatives $D^{\alpha} u(|\alpha| \leq k)$ are $L^{q}$ - 
summable over $\Omega$ is denoted as usual by $W^{k, q}(\Omega)$, and $W_{0}^{k, q}(\Omega)$ is the closure of $C_{0}^{k}(\Omega)$ with respect to the Sobolev norm $\|\cdot\|_{w^{k}, q(\Omega)}$. In what follows we adopt the standard summation convention that repeated indices mean summation from 1 to $n$.

We are aimed at the investigation of the strong solvability of the Dirichlet problem

$$
\left\{\begin{array}{l}
a^{i j}(x, u) D_{i j} u+b(x, u, D u)=0 \text { a.e. } \Omega \\
u=\varphi \text { on } \partial \Omega
\end{array}\right.
$$

where $\varphi \in W^{2, n}(\Omega)$.

As usual, by strong solution of (1.1) we mean a twice weakly differentiable function $\left(u \in W^{2, q}(\Omega), q \geq 1\right)$ that satisfies equation above almost everywhere, and $u-\varphi \in W_{0}^{1, q}(\Omega)$.

Throughout the paper we shall suppose that the functions $a^{i j}(x, z)$ and $b(x, z, p)$ are Carathéodory functions, i. e., they are measurable in $x \in \Omega$ for all $z \in \mathbf{R}, p \in \mathbf{R}^{n}$, and continuous with respect to the other variables for almost all $x \in \Omega$.

In order to state the main result, we give a list of assumptions. that

Uniform ellipticity: there exists a positive non-increasing function $\lambda(t)$ such

$$
a^{i j}(x, z) \xi^{i} \xi^{j} \geq \lambda(|z|)|\xi|^{2} \quad \text { a.a. } x \in \Omega, \forall z \in \mathbf{R}, \forall \xi \in \mathbf{R}^{n}, \quad a^{i j}=a^{j i} .
$$

Local uniform continuity of $a^{i j}$ in $z$, uniformly in $x$ :

$$
\left|a^{i j}(x, z)-a^{i j}\left(x, z^{\prime}\right)\right| \leq a(x) \mu_{M}\left(\left|z-z^{\prime}\right|\right) \text { a.e. } \Omega, \quad \forall z, \quad z^{\prime} \in[-M, M],
$$

where $a(x) \in L^{\infty}(\Omega), \mu_{M}(t)$ is non-decreasing function, and $\lim _{t \rightarrow 0} \mu_{M}(t)=$ $0, a^{i j}(x, 0) \in L^{\infty}(\Omega)$.

The coefficients $a^{i j}(x, z) \in V M O$ with respect to $x$, locally uniformly in $z$ :

$$
\sup _{\rho \leq r} \frac{1}{\left|\Omega_{\rho}\right|} \int_{\Omega_{\rho}}\left|a^{i j}(x, z)-\frac{1}{\left|\Omega_{\rho}\right|} \int_{\Omega_{\rho}} a^{i j}(y, z) d y\right| d x=\eta_{M}(r)
$$

for all $z \in[-M, M]$ and $\lim _{r \rightarrow 0} \eta_{M}(r)=0$. Here $\Omega_{\rho}$ is the intersection $\Omega \cap B_{\rho}$ with Lebesgue measure $\left|\Omega_{\rho}\right|$, where $B_{\rho}$ ranges in the class of the balls with radius $\rho$ centered at the points of $\Omega$.

As it concerns the function $b(x, z, p)$ we assume:

Quadratic growth with respect to the gradient:

$$
|b(x, z, p)| \leq \nu(|z|)\left(b_{1}(x)+|p|^{2}\right) \text { a.e. } \Omega, \quad \forall(z, p) \in \mathbf{R} \times \mathbf{R}^{n},
$$

where $\nu(t)$ is a positive non-decreasing function and $b_{1}(x) \in L^{n}(\Omega)$.

Monotonicity of $b(x, z, p)$ with respect to $z$ :

$$
\frac{\operatorname{sign} z \cdot b(x, z, p)}{\lambda(|z|)} \leq \nu_{1}(x)|p|+\nu_{2}(x) \quad \text { a. a. } x \in \Omega, \quad \forall(z, p) \in \mathbf{R} \times \mathbf{R}^{n},
$$

with $\nu_{1}, \nu_{2} \in L^{n}(\Omega), \nu_{1}, \nu_{2} \geq 0$. 
We are now in a position to formulate our existence result.

Theorem 1.1. Let $\Omega \subset \mathbf{R}^{n}$ be a bounded and $C^{1,1}$ regular domain and suppose conditions (1.2)-(1.6) to be fulfilled. Then the problem (1.1) is solvable in the strong sense, i.e. for each $\varphi \in W^{2, n}(\Omega)$ there exists a function $u \in W^{2, n}(\Omega)$ satisfying the equation in (1.1) almost everywhere, and $u-\varphi \in W_{0}^{1, n}(\Omega)$.

Using Theorem 1.1, the regularity theory for linear elliptic equations with $V M O$ coefficients due to [CFL2], as well as the usual "bootstrap" arguments, there are no difficulties in obtaining the following regularity result.

Corollary 1.2. In addition to the assumptions of Theorem 1.1 suppose $b_{1}(x) \in$ $L^{q}(\Omega)$ in (1.5), and $\varphi \in W^{2}, q(\Omega)$ for some $q>n$. Then the strong solution of the problem (1.1) belongs to the space $W^{2, q}(\Omega)$, and $u-\varphi \in W_{0}^{1, q}(\Omega)$.

Remark 1.3. 1. The Sobolev imbedding theorem ensures that the solution $u$ of (1.1) assumes continuously its boundary values $\varphi$. More precisely $u \in C^{0, \alpha}(\bar{\Omega})$ for all $\alpha \in(0,1)$, and $u \in C^{1,1-\frac{n}{q}}(\bar{\Omega})$ in the case of Corollary 1.2.

2. It follows immediately from $(1.3)$ that $a^{i j}(x, z) \in L^{\infty}(\Omega \times[-M, M])$, and the norm $\left\|a^{i j}\right\|_{L^{\infty}(\Omega \times[-M, M])}$ is bounded in terms of $M, \mu_{M},\|a\|_{L^{\infty}(\Omega)}$ and $\left\|a^{i j}(\cdot, 0)\right\|_{L^{\infty}(\Omega)}$.

3. In $\S \S 2,3$ we shall employ Chiarenza-Frasca-Longo's solvability result [CFL2] that requires the coefficients of linear elliptic equation to be in $V M O\left(\mathbf{R}^{n}\right)$. Having a function defined on $\Omega$ that is $V M O$ in sense of (1.4) it is possible to extend it to all $\mathbf{R}^{n}$ preserving the $V M O$-modulus by means of [Acq, Proposition 1.3]. In the following we will use this remark without explicit reference.

4. Assumption (1.5) on the quadratic growth of $b(x, z, p)$ with respect to the gradient is optimal for the existence result even in the case of constant coefficients, as shown by examples due to Nagumo $[\mathrm{Ng}]$ and Serrin [S].

5. Theorem 1.1 holds true in the more restrictive case of continuous in $\bar{\Omega} \times \mathbf{R}$ coefficients $a^{i j}(x, z)$. In fact, (1.3) is fulfilled with $\mu_{M}(t)$ replaced by the supremum of the moduli of continuity of $a^{i j}(x, z)$ with respect to $z$, and (1.4) is satisfied too if we put $\eta_{M}(r)$ to be the maximal $x$-continuity modulus of $a^{i j}(x, z)$.

Further, if $a^{i j}(x, z) \in W^{1, n}$ with respect to $x$, locally uniformly in $z$, then $a^{i j} \in V M O$ again ([CFL1, $\left.\left.\S 2\right]\right)$. In such a way Theorem 1.1 may be considered as a generalization of Miranda's semilinear $\left(a^{i j}=a^{i j}(x)\right)$ result (see $[\mathrm{M}]$ ) that ensures strong solvability of (1.1) assuming $a^{i j}(x) \in W^{1, n}(\Omega)$ and strictly sub-quadratic gradient growth in (1.5).

6. Monotonicity assumption (1.6) will be employed in $\S 2$ in order to derive an a priori bound for $L^{\infty}$-solution's norms. Because of the use of AleksandrovPucci's maximum principle it may be replaced by the following more general ones:

$$
\frac{\operatorname{sign} z \cdot b(x, z, p)}{\left(\operatorname{det} a^{i j}(x, z)\right)^{1 / n}} \leq \nu_{1}(x)|p|+\nu_{2}(x), \quad \nu_{1}, \nu_{2} \in L^{n}(\Omega)
$$


or even

$$
\frac{\operatorname{sign} z \cdot b(x, z, p)}{n\left(\operatorname{det} a^{i j}(x, z)\right)^{1 / n}} \leq \frac{h(x)}{g(p)}
$$

where $h \in L^{n}(\Omega), g \in L_{\text {loc }}^{n}\left(\mathbf{R}^{n}\right)$, and $\int_{\Omega} h^{n} d x<\int_{\mathbf{R}^{n}} g^{n} d p$ (see [GT, Chapter 10] for details).

Uniqueness for the Dirichlet problem (1.1) can be proved in the wider class $W_{\text {loc }}^{2, n}(\Omega) \cap C^{0}(\bar{\Omega})$ in a special case concerning the structure of the coefficients and the nonlinear term.

Theorem 1.4. Suppose the coefficients $a^{i j}$ of (1.1) to be bounded and measurable functions that are independent of $z$, and let (1.2) to hold with constant $\lambda$. Assume further that $b(x, z, p)$ is non-increasing in $z$, a.e. in $\Omega, \forall p \in \mathbf{R}^{n}$, and let

$$
\left|b(x, z, p)-b\left(x, z, p^{\prime}\right)\right| \leq b_{2}(x, z)\left|p-p^{\prime}\right|
$$

for a. a. $x \in \Omega, \forall z \in \mathbf{R}, p, p^{\prime} \in \mathbf{R}^{n}, \sup _{|z| \leq M} b_{2}(\cdot, z) \in L^{n}(\Omega)$.

If $u, v \in W_{\mathrm{loc}}^{2, n}(\Omega) \cap C^{0}(\bar{\Omega})$ solve the Dirichlet problem (1.1), then $u=v$ in $\bar{\Omega}$.

Corollary 1.5. Let $\Omega$ be $C^{1,1}$ and bounded domain, and suppose assumptions of Theorem 1.4 to be valid with (1.7) replaced by

$$
\left|b(x, z, p)-b\left(x, z, p^{\prime}\right)\right| \leq b_{3}\left(x, z, p, p^{\prime}\right)\left|p-p^{\prime}\right|
$$

where $\sup _{|z|+|p|+\left|p^{\prime}\right| \leq M} b_{3}\left(\cdot, z, p, p^{\prime}\right) \in L^{n}(\Omega)$.

Then the solution of (1.1) is unique in the space $W^{2, q}(\Omega), q>n$.

Remark 1.6. 1. It is well-known from the classical theory of quasilinear elliptic equations ([GT]) that unicity cannot be expected for (1.1) if the coefficients $a^{i j}$ depend on $z$.

2. Assumption (1.7) ensures uniqueness cannot be weakened to Hölder continuity of $b(x, z, p)$ with respect to $p$ as is shown by the next simple example.

Let $\Omega=\left\{x \in \mathbf{R}^{n}:|x|<1\right\}, \theta>1, \alpha \in(0,1)$. Then the functions $u_{1}(x)=0$ and $u_{2}(x)=1-|x|^{\theta}$ solve the Dirichlet problem

$$
\left\{\begin{array}{l}
\Delta u+\theta^{1-\alpha}(n+\theta-2)|x|^{(\theta-1)(1-\alpha)-1}|D u|^{\alpha}=0 \text { a.e. } \Omega \\
u=0 \text { on } \partial \Omega
\end{array}\right.
$$

\section{A PRIORI ESTIMATES}

This section is devoted to the establishment of all the a priori estimates needed in $\S 3$ for applying Leray-Schauder's fixed point theorem.

We start with a simple auxiliary result on composition.

Lemma 2.1. Suppose conditions (1.3) and (1.4) to be valid, and let $u(x) \in$ $C^{0}(\bar{\Omega})$. Then $a^{i j}(x, u(x)) \in V M O \cap L^{\infty}(\Omega)$ with VMO-modulus bounded in terms of the continuity modulus of $u(x)$ and $\|u\|_{L^{\infty}(\Omega)}$. 
Proof. It follows from (1.3) $a^{i j}(x, u(x)) \in L^{\infty}(\Omega) \subset B M O$. Taking an arbitrary ball $B_{\rho}$ centered at a point $x_{0} \in \Omega$ and $\rho \leq r$ we have

$$
I(\rho)=\frac{1}{\left|\Omega_{\rho}\right|} \int_{\Omega_{\rho}}\left|a^{i j}(x, u(x))-\frac{1}{\left|\Omega_{\rho}\right|} \int_{\Omega_{\rho}} a^{i j}(y, u(y)) d y\right| d x \leq I_{1}(\rho)+I_{2}(\rho),
$$

where

$$
\begin{aligned}
& I_{1}(\rho)=\frac{2}{\left|\Omega_{\rho}\right|} \int_{\Omega_{\rho}}\left|a^{i j}(x, u(x))-a^{i j}\left(x, u\left(x_{0}\right)\right)\right| d x, \\
& I_{2}(\rho)=\frac{1}{\left|\Omega_{\rho}\right|} \int_{\Omega_{\rho}}\left|a^{i j}\left(x, u\left(x_{0}\right)\right)-\frac{1}{\left|\Omega_{\rho}\right|} \int_{\Omega_{\rho}} a^{i j}\left(y, u\left(x_{0}\right)\right) d y\right| d x .
\end{aligned}
$$

Now, assumption (1.3) implies:

$$
I_{1}(\rho) \leq 2\|a\|_{L^{\infty}(\Omega)} \mu_{\|u\|_{L^{\infty}(\Omega)}}(\omega(u, r))
$$

where $\omega(u, r)$ is the modulus of continuity of $u$. On the other hand,

$$
I_{2}(\rho) \leq \eta_{\|u\|_{L^{\infty}(\Omega)}}(r)
$$

by virtue of (1.4). So

$$
\sup _{\rho \leq r} I(\rho) \leq 2\|a\|_{L^{\infty}(\Omega)} \mu_{\|u\|_{L^{\infty}(\Omega)}}(\omega(u, r))+\eta_{\|u\|_{L^{\infty}(\Omega)}}(r)
$$

and therefore $\sup _{\rho \leq r} I(\rho) \rightarrow 0$ as $r \rightarrow 0$. Thus $a^{i j}(x, u(x)) \in V M O$, and moreover the $\bar{V} M O$-modulus of the composition is bounded in terms of $\|u\|_{L^{\infty}(\Omega)}$ as well as of a monotone function of the $u$-continuity modulus.

We now aim to establish an a priori bound of $\|u\|_{W^{1,2 n(\Omega)}}$ for the strong solutions $u \in W^{2}, n(\Omega)$ of (1.1). For, we shall estimate successively $\|u\|_{L^{\infty}(\Omega)}$, the continuity modulus of $u$, and $\|D u\|_{L^{2 n}(\Omega)}$.

\subsection{A priori estimate for $\|u\|_{L^{\infty}(\Omega)}$.}

Lemma 2.2. Let $u \in W^{2, n}(\Omega)$ be a strong solution of Dirichlet's problem (1.1) and suppose conditions (1.2) and (1.6) to be satisfied. Then

$$
\|u\|_{L^{\infty}(\Omega)} \leq\|\varphi\|_{L^{\infty}(\Omega)}+C\left(n, \operatorname{diam} \Omega,\left\|\nu_{1}\right\|_{L^{n}(\Omega)}\right)\left\|\nu_{2}\right\|_{L^{n}(\Omega)} .
$$

Proof. Using the structure assumption (1.6) we have almost everywhere in the set $\Omega^{+}=\{x \in \Omega: u(x)>0\}$ :

$$
\begin{aligned}
\frac{b(x, u(x), D u(x))}{\lambda(|u(x)|)} & \leq \nu_{1}(x)|D u(x)|+\nu_{2}(x) \\
& \leq\left(\nu_{1}(x) \cdot \operatorname{sign}\left(D_{i} u(x)\right)\right) D_{i} u(x)+\nu_{2}(x) .
\end{aligned}
$$

It turns out that

$$
\bar{a}^{i j}(x) D_{i j} u+\bar{b}^{i}(x) D_{i} u \geq-\nu_{2}(x) \text { a.e. } \Omega^{+},
$$

where $\bar{a}^{i j}(x)=\frac{a^{i j}(x, u(x))}{\lambda(|u(x)|)}, \bar{b}^{i}(x)=\nu_{1}(x) \cdot \operatorname{sign}\left(D_{i} u(x)\right) \in L^{n}(\Omega)$, and $\bar{a}^{i j} \xi^{i} \xi^{j} \geq$ $|\xi|^{2}$. Bearing in mind $u \in C^{0}(\bar{\Omega})$ as consequence of Sobolev's imbedding, the 
Aleksandrov-Pucci maximum principle [GT, Chapter 9] asserts

$$
\sup _{\Omega^{+}} u \leq \sup _{\partial \Omega} u^{+}+C\left(n,\left\|\nu_{1}\right\|_{L^{n}(\Omega)}, \operatorname{diam} \Omega\right)\left\|\nu_{2}\right\|_{L^{n}(\Omega)} \quad\left(u^{+}=\max (u(x), 0)\right)
$$

that leads to the bound

$$
\sup _{\Omega} u \leq \sup _{\partial \Omega}|\varphi|+C i \mid \nu_{2} \|_{L^{n}(\Omega)} .
$$

Replacing $u$ by $-u$ in the above arguments we arrive at an estimate for $u$ from below that completes the proof of (2.1).

2.2. A priori estimate for the modulus of continuity of $u$. We shall derive later an estimate for the $L^{2 n}(\Omega)$-norm of the gradient $D u$ based on the $W^{2, q}$ estimates for linear elliptic equations with $V M O$ coefficients [CFL2]. For, we need to control the $V M O$-moduli of the composition $a^{i j}(x, u(x))$. Lemma 2.1 reduces this problem to an a priori bound for the continuity modulus of the solution $u$ of (1.1). On the other hand the function $u(x)$ solves an uniformly elliptic equation, and having in mind the growth condition (1.5) it is easy to derive an a priori bound for some Hölder norm of $u(x)$ that automatically provides the desired control on the modulus of continuity. This bound for the Hölder norm is obtained by the technique due to Krylov and Safonov which generalizes classical estimates of De Giorgi-Nash-Moser, and it is based on the weak Harnack inequalities.

Lemma 2.3. Let the assumptions (1.2), (1.3) and (1.5) be fulfilled. If $u \in$ $W^{2, n}(\Omega), u-\varphi \in W_{0}^{1, n}(\Omega)$, is a strong solution of the problem (1.1) then

$$
\underset{B_{\rho} \cap \Omega}{\operatorname{osc}} u \leq C \rho^{\alpha}
$$

for each ball $B_{\rho}$ with radius $\rho$, where $\alpha<1$ and $C$ are positive constants, depending on $n, \Omega, \mu, \nu, \lambda,\|a\|_{L^{\infty}(\Omega)},\left\|a^{i j}(\cdot, 0)\right\|_{L^{\infty}(\Omega)},\left\|b_{1}\right\|_{L^{n}(\Omega)},\|\varphi\|_{W^{2}, n_{(\Omega)}}$, and $\|u\|_{L^{\infty}(\Omega)}$.

Proof. See [GT, Lemma 15.4] for the interior Hölder bound. The boundary estimate follows in the same manner using the boundary Harnack inequality [GT, Theorem 9.27]. What we need in addition is information about $\operatorname{osc}_{\partial \Omega} u$ that follows from Morrey's lemma, $u=\varphi$ on $\partial \Omega, \varphi \in W^{2, n}$, and $\partial \Omega \in$ $C^{1,1}$.

2.3. A priori estimate for $L^{2 n}(\Omega)$ norm of the gradient $D u$. The crucial step in proving our existence result is ensured by the possibility to derive an a priori bound for $\|D u\|_{L^{2 n(\Omega)}}$ in terms of $\|u\|_{L^{\infty}(\Omega)}$. The method used here is inspired by Amann-Crandall's [AC] approach in obtaining $L^{\infty}$ gradient estimate for semilinear equations with continuous coefficients.

Lemma 2.4. Assume conditions (1.2), (1.3), (1.4) and (1.5) to be fulfilled. Then

$$
\|D u\|_{L^{2 n(\Omega)}} \leq C
$$

for all solutions $u \in W^{2, n}(\Omega)$ of (1.1), where the constant $C$ depends on $n$, $\Omega, \mu, \nu, \lambda,\|a\|_{L^{\infty}(\Omega)},\left\|b_{1}\right\|_{L^{n}(\Omega)},\|\varphi\|_{w^{2}, n^{n}(\Omega)}$, and $\|u\|_{L^{\infty}(\Omega)}$. 
Proof. The equation in (1.1) is equivalent to

$$
a^{i j}(x, u) D_{i j} u+\frac{b(x, u, D u)}{b_{1}(x)+|D u|^{2}}\left(b_{1}(x)+|D u|^{2}\right)-b_{1}(x) u=-b_{1}(x) u(x) .
$$

So $u \in W^{2, n}(\Omega)$ solves

$$
A^{i j}(x) D_{i j} u+B(x)|D u|^{2}-b_{1}(x) u(x)=C(x)
$$

where

$$
\left\{\begin{aligned}
A^{i j}(x) & =a^{i j}(x, u(x)) \in V M O \cap L^{\infty}(\Omega) \quad(\text { Lemma 2.1), } \\
B(x) & =\frac{b(x, u(x), D u(x))}{b_{1}(x)+|D u|^{2}} \in L^{\infty}(\Omega), \\
C(x) & =-b_{1}(x) u(x)-\frac{b_{1}(x) b(x, u(x), D u(x))}{b_{1}(x)+|D u|^{2}} \in L^{n}(\Omega) .
\end{aligned}\right.
$$

Fixing the solution $u(x)$ of (1.1) we consider now (cf. [AC]) the one-parameter-family of Dirichlet problems

$$
\left\{\begin{array}{l}
A^{i j}(x) D_{i j} v_{\sigma}+B(x)\left|D v_{\sigma}\right|^{2}-b_{1}(x) v_{\sigma}=\sigma C(x), \\
v_{\sigma}=\sigma \varphi \text { on } \partial \Omega
\end{array}\right.
$$

with solutions $v_{\sigma}(x) \in W^{2, n}(\Omega), v_{\sigma}-\sigma \varphi \in W_{0}^{1, n}(\Omega)$ for $\sigma \in[0,1]$.

What we know about (2.4) at this moment is that $v=0$, solves (2.4) if $\sigma=0$, and solution $u(x)$ of (1.1) satisfies (2.4) if $\sigma=1$. The strategy we will follow is to estimate $\left\|D v_{\sigma_{2}}\right\|_{L^{2 n}(\Omega)}$ in terms of $\left\|D v_{\sigma_{1}}\right\|_{L^{2 n}(\Omega)}$ assuming $\sigma_{2}-\sigma_{1}>0$ to be sufficiently small. Then if the problem (2.4) is uniquely solvable for each value $\sigma \in[0,1]$ it will be possible to derive the estimate (2.2) after iteration of $W^{1,2 n}(\Omega)$-norms of $v_{\sigma}, \sigma<1$.

Proposition 2.5. Let $v_{\sigma_{1}}, v_{\sigma_{2}} \in W^{2, n}(\Omega)$ solve (2.4) for $\sigma_{1} \leq \sigma_{2}$. Then (2.5) $\left\|v_{\sigma_{1}}-v_{\sigma_{2}}\right\|_{L^{\infty}(\Omega)} \leq\left(\sigma_{2}-\sigma_{1}\right)\left(\nu_{0}+\|u\|_{L^{\infty}(\Omega)}\right), \quad \nu_{0}=\nu\left(\|u\|_{L^{\infty}(\Omega)}\right)$. Proof. Putting $w=v_{\sigma_{1}}-v_{\sigma_{2}}$ and defining the linear elliptic operator

$$
\mathscr{L} \equiv A^{i j}(x) D_{i j}+B^{i}(x) D_{i}-b_{1}(x),
$$

with

we have

$$
B^{i}(x)=2 B(x) \int_{0}^{1}\left(\theta D_{i} w+D_{i} v_{\sigma_{2}}\right) d \theta \in L^{n}(\Omega)
$$

$$
\mathscr{L} w=A^{i j}(x) D_{i j} w+B^{i}(x) D_{i} w-b_{1}(x) w=\left(\sigma_{1}-\sigma_{2}\right) C(x) \quad \text { a.e. } \Omega .
$$

Now

$$
\begin{aligned}
\left(\sigma_{1}-\sigma_{2}\right) C(x) & \geq\left(\sigma_{2}-\sigma_{1}\right)\left(-b_{1}(x)\|u\|_{L^{\infty}(\Omega)}-b_{1}(x) \nu_{0}\right) \\
& =\mathscr{L}\left(\left(\sigma_{2}-\sigma_{1}\right)\left(\nu_{0}+\|u\|_{L^{\infty}(\Omega)}\right)\right)
\end{aligned}
$$

by virtue of (1.5). Thus

$$
\mathscr{L}\left(w(x)-\left(\sigma_{2}-\sigma_{1}\right)\left(\nu_{0}+\|u\|_{L^{\infty}(\Omega)}\right)\right) \geq 0 \text { a.e. } \Omega
$$


and $w(x)=\left(\sigma_{1}-\sigma_{2}\right) \varphi(x) \leq\left(\sigma_{2}-\sigma_{1}\right)\left(\nu_{0}+\|u\|_{L^{\infty}(\Omega)}\right)$ on $\partial \Omega$. It follows from maximum principle (recall $0<b_{1}(x) \in L^{n}(\Omega)$ )

$$
w(x) \leq\left(\sigma_{2}-\sigma_{1}\right)\left(\nu_{0}+\|u\|_{L^{\infty}(\Omega)}\right) \quad \text { in } \Omega .
$$

In the same way

$$
\mathscr{L}\left(w(x)+\left(\sigma_{2}-\sigma_{1}\right)\left(\nu_{0}+\|u\|_{L^{\infty}(\Omega)}\right)\right) \leq 0 \text { a.e. } \Omega
$$

and $w(x) \geq-\left(\sigma_{2}-\sigma_{1}\right)\left(\nu_{0}+\|u\|_{L^{\infty}(\Omega)}\right)$ on $\partial \Omega$, that implies

$$
w(x) \geq-\left(\sigma_{2}-\sigma_{1}\right)\left(\nu_{0}+\|u\|_{L^{\infty}(\Omega)}\right) \quad \text { in } \Omega .
$$

Thus

$$
\|w\|_{L^{\infty}(\Omega)}=\left\|v_{\sigma_{1}}-v_{\sigma_{2}}\right\|_{L^{\infty}(\Omega)} \leq\left(\sigma_{2}-\sigma_{1}\right)\left(\nu_{0}+\|u\|_{L^{\infty}(\Omega)}\right) .
$$

Remark 2.6. In the special case $\sigma_{1}=\sigma_{2}$ the estimate (2.5) provides an uniqueness result for (2.4) for each fixed $\sigma$.

Now, let $\sigma_{1}<\sigma_{2}$ be two arbitrary numbers and suppose there exist solutions $v_{\sigma_{1}}$ and $v_{\sigma_{2}}$ of (2.4). The difference $w=v_{\sigma_{1}}-v_{\sigma_{2}} \in W^{2, n}(\Omega)$ solves

$$
\left\{\begin{array}{l}
A^{i j}(x) D_{i j} w=\left(\sigma_{1}-\sigma_{2}\right) C(x)-B(x)\left(\left|D v_{\sigma_{1}}\right|^{2}-\left|D v_{\sigma_{2}}\right|^{2}\right)+b_{1}(x) w \quad \text { a.e. } \Omega \\
w=\left(\sigma_{1}-\sigma_{2}\right) \varphi \text { on } \partial \Omega .
\end{array}\right.
$$

It follows ([CFL2, Theorem 4.2])

$$
\begin{array}{r}
\|w\|_{W^{2}, n(\Omega)} \leq C\left(\left\|\left(\sigma_{1}-\sigma_{2}\right) C(x)-B(x)\left(\left|D v_{\sigma_{1}}\right|^{2}-\left|D v_{\sigma_{2}}\right|^{2}\right)+b_{1}(x) w\right\|_{L^{n}(\Omega)}\right. \\
\left.+\|\varphi\|_{W^{2, n}(\Omega)}+\|w\|_{L^{\infty}(\Omega)}\right) .
\end{array}
$$

We note that the constant $C$ above depends on $n, \lambda, \mu, \nu,\|a\|_{L^{\infty}(\Omega)}$, $\left\|a^{i j}(\cdot, 0)\right\|_{L^{\infty}(\Omega)}, \Omega,\|u\|_{L^{\infty}(\Omega)}$, and the $V M O$-moduli of $A^{i j}(x)$. On the other hand Lemma 2.1 and Lemma 2.3 show that the dependence of the VMOmodulus of $A^{i j}(x)$ on $u$ is through $\|u\|_{L^{\infty}(\Omega)}$ only. Thus using representations (2.3), as well as estimate (2.5) for $\|w\|_{L^{\infty}(\Omega)}$ we obtain

$$
\|w\|_{w^{\cdot 2, n}(\Omega)} \leq C\left(1+\|D w\|_{L^{2 n}(\Omega)}^{2}+\left\|D v_{\sigma_{1}}\right\|_{L^{2 n}(\Omega)}^{2}\right)
$$

and the new constant $C$ does not depend on $\sigma_{1}-\sigma_{2}$.

Application of Gagliardo-Nirenberg's interpolation inequality [N, p. 125] and (2.5) leads to

$$
\|D w\|_{L^{2 n}(\Omega)}^{2} \leq K\left\|D^{2} w\right\|_{L^{n}(\Omega)} \cdot\|w\|_{L^{\infty}(\Omega)} \leq K\left(\sigma_{2}-\sigma_{1}\right)\left(\nu_{0}+\|u\|_{L^{\infty}(\Omega)}\right)\left\|D^{2} w\right\|_{L^{n}(\Omega)}
$$

(the constant $K$ depends on $n, \Omega$ but not on $w$ ), and utilizing (2.6) we arrive at

$$
\|D w\|_{L^{2 n}(\Omega)}^{2} \leq C\left(1+\left(\sigma_{2}-\sigma_{1}\right)\|D w\|_{L^{2 n}(\Omega)}^{2}+\left\|D v_{\sigma_{1}}\right\|_{L^{2 n}(\Omega)}^{2}\right)
$$

where $C$ is independent of $\sigma_{i}$. 
If we assume now $\sigma_{2}-\sigma_{1} \leq \delta$ with $C . \delta<\frac{1}{2}$ we have

$$
\left\|D v_{\sigma_{2}}\right\|_{L^{2 n}(\Omega)}^{2} \leq C^{\prime}+C^{\prime \prime}\left\|D v_{\sigma_{1}}\right\|_{L^{2 n}(\Omega)}^{2}
$$

(recall $w=v_{\sigma_{1}}-v_{\sigma_{2}}$ ) whenever $\sigma_{2}-\sigma_{1} \leq \delta$. Here the constants $C^{\prime}$ and $C^{\prime \prime}$ depend on $n, \lambda, \mu, \nu, \Omega,\|u\|_{L^{\infty}(\Omega)}$, and $\|\varphi\|_{W^{2}, n_{(\Omega)}}$.

In particular, if we put $\sigma_{1}=0$ and $\sigma_{2}=\delta$ above, the uniqueness assertion (Remark 2.6) and (2.7) show

$$
\left\|D v_{\delta}\right\|_{L^{2 n}(\Omega)}^{2} \leq C^{\prime}
$$

whenever there exists the solution $v_{\delta} \in W^{2, n}(\Omega)$ of (2.4) with $\sigma=\delta$.

To prove solvability of Dirichlet's problem (2.4) for $\sigma=\delta$ Leray-Schauder's theorem will be employed. For this we define an operator $\mathscr{F}$ whose fixed point solves (2.4) when $\sigma=\delta$. Taking $w \in W^{1,2 n}(\Omega)$ we put $v=\mathscr{F} w \in W^{2, n}(\Omega)$ to be the unique solution of the linear Dirichlet problem

$$
\left\{\begin{array}{l}
A^{i j}(x) D_{i j} v=\delta C(x)-B(x)|D w|^{2}+b_{1}(x) w \\
v=\delta \varphi \text { on } \partial \Omega
\end{array}\right.
$$

Indeed, the last problem is solvable by means of (2.3) and [CFL2, Theorem 4.4]. Moreover, Chiarenza-Frasca-Longo's result asserts that $\mathscr{F}$ is a continuous operator, and because $W^{2}, n \hookrightarrow W^{1,2 n}$ compactly it is also a compact one when considered as a mapping from $W^{1,2 n}$ into itself. Further, the estimate (2.8) provides an a priori bound for each solution of the equation $v=\tau \mathscr{F} v, \tau \in[0,1]$, that is equivalent to

$$
\left\{\begin{array}{l}
A^{i j}(x) D_{i j} v=\tau\left(\delta C(x)-B(x)|D v|^{2}+b_{1}(x) v\right), \\
v=\tau \delta \varphi \text { on } \partial \Omega
\end{array}\right.
$$

Thus Leray-Schauder's theorem [GT, Theorem 11.3] ensures the existence of a fixed point of the mapping $\mathscr{F}$ that proves solvability of the Dirichlet problem (2.4) in the case $\sigma=\delta$.

Taking now $\sigma_{1}=N \delta, \sigma_{2}=(N+1) \delta, N=1,2, \ldots$, in (2.7) and using finitely many times the above procedure we can estimate $\|u\|_{W^{1}, 2 n(\Omega)}=$ $\left\|v_{\{\sigma=1\}}\right\|_{W^{1}, 2 n(\Omega)}$ in terms of known quantities. This proves Lemma 2.4 .

Combining Lemma 2.2 and Lemma 2.4 with the global estimate of [CFL2, Theorem 4.2] we obtain the following a priori bound.

Corollary 2.7. Let $\Omega$ be $C^{1,1}$ bounded domain, and suppose conditions (1.2)(1.6) to be fulfilled with $b_{1}(x) \in L^{q}(\Omega)$ in (1.5), $q \geq n$. If $\varphi \in W^{2}, q(\Omega)$, then there exists an absolute constant $C$ such that

$$
\|u\|_{W^{2, q(\Omega)}} \leq C
$$

for each solution $u \in W^{2, q}(\Omega)$ of the Dirichlet problem (1.1).

\section{Proofs of the theOREMS}

We shall use Leray-Schauder's principle again in order to prove Theorem 1.1. For this let us fix $v \in W^{1,2 n}(\Omega)$. Then $v(x)$ is Hölder continuous in $\bar{\Omega}$ 
with exponent $1 / 2$ by Morrey's lemma. It follows from Lemma 2.1 that $a^{i j}(x, v(x))$ are $V M O \cap L^{\infty}(\Omega)$ functions. Furthermore, assumption (1.5) ensures $b(x, v(x), D v(x)) \in L^{n}(\Omega)$. Applying now an existence result for linear elliptic equations with $V M O$-coefficients ([CFL2, Theorem 4.4]) we conclude the existence of a unique strong solution $u(x) \in W^{2, n}(\Omega)$ of the linear Dirichlet problem:

$$
\left\{\begin{array}{l}
a^{i j}(x, v(x)) D_{i j} u=-b(x, v(x), D v(x)) \quad \text { a.e. } \Omega \\
u=\varphi \quad \text { on } \partial \Omega
\end{array}\right.
$$

for arbitrary $\varphi \in W^{2, n}(\Omega)$. Proceeding as in $\S 2$ and bearing in mind Sobolev's imbedding $W^{2, n}(\Omega) \hookrightarrow W^{1,2 n}(\Omega)$ we define a compact nonlinear mapping

$$
\mathscr{F}: W^{1,2 n}(\Omega) \longrightarrow W^{1,2 n}(\Omega)
$$

by $\mathscr{F} v=u$. It is clear that each fixed point of $\mathscr{F}$ will be a solution of the Dirichlet problem (1.1). To prove continuity of $\mathscr{F}$ we put $u_{h}=\mathscr{F} v_{h}, u=$ $\mathscr{F} v$, where $v_{h} \rightarrow v$ in $W^{1,2 n}(\Omega)$ as $h \rightarrow 0$. The operator $\mathscr{F}$ maps bounded sets in $W^{1,2 n}(\Omega)$ into bounded sets in $W^{2, n}(\Omega)$ by virtue of Lemma 2.1, linear estimate ([CFL2, Theorem 4.2]) and maximum principle. Moreover, the difference $u_{h}-u \in W^{2, n}(\Omega) \cap W_{0}^{1, n}(\Omega)$ satisfies

$$
\begin{aligned}
\left\|u_{h}-u\right\|_{W^{2}, n_{(\Omega)}} \leq C & \left\|b\left(x, v_{h}, D v_{h}\right)-b(x, v, D v)\right\|_{L^{n}(\Omega)} \\
& \left.+\left\|D^{2} u_{h}\left(a^{i j}\left(x, v_{h}\right)-a^{i j}(x, v)\right)\right\|_{L^{n}(\Omega)}\right) .
\end{aligned}
$$

The first term in the right-hand side above tends to 0 as $h \rightarrow 0$ by means of (1.5) and continuity of the Nemytskii operator defined by $b$ ([FK, Theorem 16.11]). The same conclusion is valid for the second term because of the boundedness of $\left\|D^{2} u_{h}\right\|_{L^{n}(\Omega)},(1.3)$ and $\left\|v_{h}-v\right\|_{L^{\infty}(\Omega)} \rightarrow 0$. Thus $u_{h} \rightarrow u$ in $W^{2, n}(\Omega)$ that proves the continuity of $\mathscr{F}$. To assert the existence of a fixed point of $\mathscr{F}$, i. e. of a solution of (1.1), it remains to prove the a priori estimate

$$
\|u\|_{W^{1,2 n(\Omega)}} \leq C
$$

with a constant $C$ independent of $u$ and $\tau$, for each solution $u$ of the problem

$$
\left\{\begin{array}{l}
a^{i j}(x, u) D_{i j} u+\tau b(x, u, D u)=0 \quad \text { a.e. } \Omega \\
u=\tau \varphi \quad \text { on } \partial \Omega .
\end{array}\right.
$$

The desired estimate (3.1), however, is a direct consequence of Lemma 2.4. This completes the proof of Theorem 1.1.

Proof of Theorem 1.4. The difference $w=u-v \in W_{\mathrm{loc}}^{2, n}(\Omega) \cap C^{0}(\bar{\Omega})$ solves

$$
\begin{aligned}
a^{i j}(x) D_{i j} w & +b(x, u, D u)-b(x, u, D v) \\
& +b(x, u, D v)-b(x, v, D v)=0 \text { a.e. } \Omega .
\end{aligned}
$$

Putting $\Omega^{+}=\{x \in \Omega: w(x)>0\}$, we have

$$
b(x, u(x), D v(x))-b(x, v(x), D v(x)) \leq 0 \text { a.e. } \Omega^{+}
$$


$(b(x, z, p)$ is non-increasing in $z)$, and

$$
\begin{aligned}
b(x, u(x), D u(x)) & -b(x, u(x), D v(x)) \leq b_{2}(x, u(x))|D w(x)| \\
& \leq \sum_{i=1}^{n}\left(b_{2}(x, u(x)) \cdot \operatorname{sign} D_{i} w(x)\right) D_{i} w(x)=b^{i}(x) D_{i} w(x)
\end{aligned}
$$

where $b^{i}(x)=b_{2}(x, u(x)) \cdot \operatorname{sign} D_{i} w(x) \in L^{n}(\Omega)$ as consequence of (1.7).

Thus (3.2) leads to

$$
a^{i j}(x) D_{i j} w+b^{i}(x) D_{i} w \geq 0 \quad \text { a.e. } \Omega^{+} .
$$

Applying the Aleksandrov-Pucci maximum principle we obtain

$$
w(x) \leq \sup _{\Omega^{+}} w \leq \sup _{\partial \Omega^{+}} w^{+}=0 \quad(w=0 \text { on } \partial \Omega)
$$

that shows $w \leq 0$ in $\bar{\Omega}$. Analogously, replacing $w$ by $-w$ above we have $w \geq 0$, i.e. $u=v$ in $\bar{\Omega}$.

Corollary 1.5 can be proved in the same fashion. Indeed $u, v \in W^{2, q}(\Omega) \subset$ $C^{1,1-\frac{n}{q}(\bar{\Omega})}\left(\partial \Omega \in C^{1,1}\right)$ now, and $b^{i}(x)=b_{3}(x, u(x), D u(x), D v(x))$. $\operatorname{sign} D_{i} w(x) \in L^{n}(\Omega)$ again, by virtue of $\left(1.7^{\prime}\right)$.

\section{ACKNOWLEDGEMENTS}

The results presented here were obtained during authors's stay at Università degli studi di Catania as C.N.R.-G.N.A.F.A. fellow. The author wishes to express his deep gratitude to the Italian National Research Council (C.N.R.) for the support, to Professor Antonino Maugeri for pointing out to his attention Miranda's result, as well as to Filippo Chiarenza for the encouragement and the good discussions we had with him.

\section{REFERENCES}

[AC] H. Amann and M. Crandall, On some existence theorems for semi-linear elliptic equations, Indiana Univ. Math. J. 27 (1978), 779-790.

[Acq] P. Acquistapace, On BMO regularity for linear elliptic systems, Ann. Mat. Pura Appl. 161 (1992), 231-270.

[CFL1] F. Chiarenza, M. Frasca and P. Longo, Interior $W^{2, p}$ estimates for non divergence elliptic equations with discontinuous coefficients, Ricerche Mat. 60 (1991), 149-168.

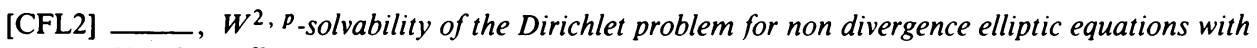
VMO coefficients, Trans. Amer. Math. Soc. 336 (1993), 841-853.

[FK] S. Fučik and A. Kufner, Nonlinear differential equations, Elsevier, New York, 1980.

[GT] D. Gilbarg and N. S. Trudinger, Elliptic partial differential equations of second order, 2nd ed., Springer-Verlag, Berlin, 1983.

[L] G. M. Lieberman, Gradient estimates for semilinear elliptic equations, Proc. Royal Soc. Edinburgh 100A (1985), 11-17.

[M] C. Miranda, Sulle equazioni ellittiche del secondo ordine di tipo non variazionale, a coefficienti discontinui, Ann. Mat. Pura Appl. 63 (1963), 353-386.

[N] L. Nirenberg, On elliptic partial differential equations, Ann. Scuola Norm. Sup. Pisa (3) 13 (1959), 115-162.

[Ng] M. Nagumo, On principally linear elliptic differential equations of the second oraer, Osaka Math. J. 6 (1954), 207-229. 
[S] J. Serrin, The problem of Dirichlet for quasilinear elliptic differential equations with many independent variables, Philos. Trans. Roy. Soc. London, Ser. A 264 (1969), 413-496.

[To] F. Tomi, Über semilineare elliptische Differentialgleichungen zweiter Ordnung, Math. Z. 111 (1969), 350-366.

[Tr] G. M. Troianiello, Maximal and minimal subsolutions to a class of eliiptic quasilinear problems, Proc. Amer. Math. Soc. 91 (1984), 95-102.

Department of Mathematics, Technological University of Sofia, 8 "Kl. OkhridSKI" BLV., SOFIA 1156, BulgaRIA

E-mail address: dian@bgcict.bitnet 